\title{
Brain perfusion abnormalities in Alzheimer's disease: comparison between patients with focal temporal lobe dysfunction and patients with diffuse cognitive impairment
}

\author{
A Cappa, M L Calcagni, G Villa, A Giordano, C Marra, G De Rossi, M Puopolo, \\ G Gainotti
}

\begin{abstract}
Objectives-Patients with Alzheimer's disease (AD) showing a selective impairment of episodic and semantic memory have recently been classified as affected by focal temporal lobe dysfunction (FTLD) and considered as a distinct subgroup of patients affected by a particular form of AD. The aim was to compare the cerebral perfusion of patients with AD with FTLD and patients with $A D$ with the more typical profile of diffuse cognitive impairment (dAD).

Methods-Ten patients with AD with
\end{abstract} FTLD, 14 patients with $A D$ with $\mathrm{dAD}$, and 12 normal controls were studied. All the 24 patients with $A D$ underwent a complete neuropsychological assessment. SPECT examination with [ $\left.{ }^{99_{\mathrm{m}}} \mathrm{Tc}\right]-\mathrm{HMPAO}$, using a four head brain dedicated tomograph, was performed in patients and controls. Tracer uptake was quantified in 27 regions of interest (ROIs), including lateral and mesial temporal areas. Mean counts in the 27 ROIs of controls, patients with FTLD and those with dAD were compared using an ANOVA for repeated measures with Bonferroni's correction. A logistic regression analysis, followed by a receiver operating characteristic (ROC) analysis, was also applied to select SPECT patterns which significantly differentiated patients with FTLD and those with dAD.

Results-Two scintigraphic patterns of abnormalities, shaping a double dissociation between the FTLD and dAD groups, emerged: a bilateral mesial temporal hypoperfusion, characteristic of FTLD and a posterior parietal (and temporal parietal) hypoperfusion characteristic of patients with dAD.

Conclusions-These scintigraphic findings provide further support to the hypothesis that FTLD is not a mere stage but a distinct anatomoclinical form of AD. The combination of neuropsychological tests and $\left[{ }^{99 \mathrm{~m}} \mathrm{Tc}\right]-H M P A O$ SPECT may be very useful in identifying patients with FTLD from the wider group of patients with dAD. This issue is particularly worthwhile, as there is increasing evidence that patients with FTLD have a slower rate of cognitive decline. (f Neurol Neurosurg Psychiatry 2001;70:22-27)
Keywords: Alzheimer's disease; focal temporal lobe dysfunction; neuropsychological profiles; [ $\left.{ }^{99 \mathrm{~m}} \mathrm{Tc}\right]-$ HMPAO SPECT

Alzheimer's disease (AD) is usually characterised by a diffuse or plurisegmental cognitive impairment with a consistent deterioration of memory. This cognitive pattern is often seen in the advanced stages of dementia. Early in the course of the disease, neuropsychological assessment generally shows deterioration of memory associated with loss of at least one other cognitive ability. ${ }^{1}$

Studies on cognitive performance of patients with early $\mathrm{AD}$ showed the existence of different subgroups of patients who present different neuropsychological profiles, reflecting different qualitative patterns of cognitive impairment. ${ }^{2}$ Usually, there is a close relation between neuropsychological profiles and patterns of regional brain dysfunction as shown by PET or SPECT. ${ }^{34}$

More recently, Butters et $a \bar{l}$ identified a subgroup of patients with $\mathrm{AD}$ who showed selective deficits in verbal and non-verbal memory, naming, and word generation. They attributed these deficits to a bilateral dysfunction of the temporal lobe and therefore labelled this subgroup as having focal temporal lobe dysfunction (FTLD). The same authors also reported that, compared with patients with $\mathrm{AD}$ with a more diffuse pattern of cognitive impairment (dAD), patients with FTLD presented a slower rate of cognitive decline, with preservation of executive functions. They concluded that the FTLD group was not a mere group of patients in the early stages of the more common form of $\mathrm{dAD}$ but represented a distinct subgroup of patients probably affected by a biological variant of AD.

In this study we performed cerebral [ $\left.{ }^{99 \mathrm{~m}} \mathrm{Tc}\right]-$ HMPAO SPECT in patients with AD with FTLD and in patients with AD with a diffuse pattern of cognitive deficits (dAD) to verify whether the two groups could be differentiated not only on the basis of their neuropsychological profiles but also considering the scintigraphic patterns of cerebral perfusion abnormalities.

\section{Methods}

PATIENTS

Fourteen patients with dAD, 10 patients with FTLD, and 12 control subjects were studied. All the 24 patients ( $14 \mathrm{dAD}+10$ FTLD) were 
Table 1 Mental deterioration battery (MDB): mean scores (SD) obtained by patients with FTLD and those with $d A D$

\begin{tabular}{lrrr}
\hline & FTLD $(n=10)$ & dAD (n=14) & Cut off* \\
\hline Visual memory & $14.6(3.1)$ & $13.6(3.3)$ & 13.8 \\
Rey’s auditory verbal learning test & & & \\
$\quad$ Immediate recall & $20.8(3.7)$ & $17.1(5.9)$ & 28.5 \\
$\quad$ Delayed recall & $1.2(0.9)$ & $2.1(1.9)$ & 4.6 \\
$\quad$ Delayed recognition $\dagger$ & $73.3(8.0)$ & $82.9(7.7)$ & 89.6 \\
Phonological verbal fluency & $20.8(8.8)$ & $13.8(7.8)$ & 17.3 \\
Word generation (semantic categories) & $8.7(4.2)$ & $8.1(5.5)$ & 15.5 \\
Copying drawings & $7.9(3.6)$ & $5.9(3.0)$ & 7.2 \\
Copying drawings with landmarks $\ddagger$ & $63.1(8.9)$ & $51.6(7.5)$ & 61.8 \\
Digit span forward & $5.8(1.7)$ & $5.0(0.9)$ & 7.3 \\
Digit span backward & $3.1(1.1)$ & $2.1(1.3)$ & 5.2 \\
Raven’s coloured progressive matrices & $25.4(5.4)$ & $14.8(4.5)$ & 18.9 \\
Temporal rule induction\} $&{22.3(5.4)} &{17.8(4.9)} &{15.7} \\
{\hline}$
\end{tabular}

${ }^{\star}$ Cut off values derived from the validation $\operatorname{study}^{7}$ of the MDB, based on the analysis of performance of 340 normal subjects.

$\dagger \mathrm{McNicol}$ 's accuracy scores (signal detection theory) range 50-100 (random performance errorless performance).

$\ddagger$ Visuoconstructive tasks

§Task exploring "sequencing" and "set shifting", most sensitive to frontal damage.

drawn from an unselected consecutive series of patients referred to our neuropsychological service for a suspicion of dementia. They had received the diagnosis of probable $\mathrm{AD}$ by a senior staff neurologist, according to the DSM-III-R diagnostic criteria for primary degenerative dementia and the NINCDSADRDA criteria for probable AD. All the 24 patients presented with progressive cognitive impairment-consistently affecting memory and less consistently affecting semantic and lexical abilities, visuospatial functions, and executive functions - with a history of between 8 and 24 months since the beginning of symptoms. In all cases there was a decline in performance affecting two or more areas of cognition, as assessed at preliminary neuropsychological examination by the mini mental state examination (MMSE) ${ }^{6}$ and the mental deterioration battery (MDB), ${ }^{7}$ a standardised set of neuropsychological tests commonly used

Table 2 Mean counts (SD) in the 27 ROIs of controls, and patients with FTLD and those with $d A D$

\begin{tabular}{|c|c|c|c|}
\hline ROIs & $C S(n=2)$ & $F T L D(n=10)$ & $d A D(n=14)$ \\
\hline Anterior temporal right & $0.671(0.098)$ & $0.547(0.089)$ & $0.649(0.100)$ \\
\hline Anterior temporal left & $0.674(0.106)$ & $0.504(0.094)^{\star}$ & $0.588(0.102)$ \\
\hline Pons & $0.784(0.069)$ & $0.733(0.092)$ & $0.726(0.76)$ \\
\hline Emicerebellar right & $0.996(0.007)$ & $1(0)$ & $0.987(0.18)$ \\
\hline Emicerebellar left & $0.968(0.034)$ & $0.953(0.020)$ & $0.971(0.037)$ \\
\hline Anterior frontal right & $0.735(0.060)$ & $0.701(0.079)$ & $0.721(0.048)$ \\
\hline Anterior frontal left & $0.717(0.050)$ & $0.680(0.064)$ & $0.709(0.057)$ \\
\hline Middle frontal right & $0.743(0.039)$ & $0.714(0.085)$ & $0.733(0.54)$ \\
\hline Middle frontal left & $0.738(0.048)$ & $0.699(0.077)$ & $0.706(0.062)$ \\
\hline Sylvian right & $0.752(0.045)$ & $0.749(0.085)$ & $0.715(0.79)$ \\
\hline Sylvian left & $0.726(0.041)$ & $0.687(0.079)$ & $0.684(0.079)$ \\
\hline Temporal-parietal right & $0.742(0.049)$ & $0.715(0.074)$ & $0.634(0.126) \dagger$ \\
\hline Temporal-parietal left & $0.708(0.055)$ & $0.670(0.061)$ & $0.586(0.086)+t+\$$ \\
\hline Occipital right & $0.775(0.051)$ & $0.743(0.064)$ & $0.701(0.119)$ \\
\hline Occipital left & $0.775(0.053)$ & $0.715(0.065)$ & $0.664(0.105) \dagger$ \\
\hline Thalamus right & $0.726(0.100)$ & $0.645(0.102)$ & $0.657(0.096)$ \\
\hline Thalamus left & $0.689(0.129)$ & $0.627(0.084)$ & $0.646(0.100)$ \\
\hline Superior frontal right & $0.753(0.063)$ & $0.696(0.060)$ & $0.650(0.118) \dagger$ \\
\hline Superior frontal left & $0.721(0.049)$ & $0.657(0.066)$ & $0.613(0.115) \dagger$ \\
\hline Central right & $0.753(0.050)$ & $0.700(0.049)$ & $0.645(0.125) \dagger$ \\
\hline Central left & $0.744(0.034)$ & $0.688(0.047)^{\star}$ & $0.630(0.124) \dagger$ \\
\hline Parietal right & $0.761(0.056)$ & $0.743(0.085)$ & $0.600(0.133)+t+s$ \\
\hline Parietal left & $0.784(0.050)$ & $0.720(0.071)$ & $0.604(0.126)+t+\Phi$ \\
\hline Mesial temporal right & $0.832(0.324)$ & $0.476(0.079)^{\star}$ & $0.630(0.122) \$$ \\
\hline Mesial temporal left & $0.862(0.390)$ & $0.432(0.087)^{\star \star \star}$ & $0.571(0.110) \Phi$ \\
\hline Lateral temporal right & $0.918(0.402)$ & $0.648(0.099)$ & $0.668(0.106)$ \\
\hline Lateral temporal left & $0.934(445)$ & $0.571(0.113)^{\star}$ & $0.605(0.110)$ \\
\hline
\end{tabular}

Student's $t$ test with Bonferroni's correction for multiple comparisons:

Significant differences:

FTLD $v$ CS ${ }^{\star} \mathrm{p}<0.0167 ;{ }^{\star \star \star}{ }^{\star} \mathrm{p}<0.0006$.

$\mathrm{dAD} v \mathrm{CS}+\mathrm{p}<0.0167 ;$ tttp $<0.0006$.

FTLD $v$ dAD $\$ \mathrm{p}<0.0167$. in our service as a screening device for the assessment of intellectual deterioration. All the 24 patients had a Hachinski's ischaemia scale score less than 4 . None of them had a history of familial AD. Patients were excluded from the study if they had: (1) a history of alcohol or drug misuse and of head trauma; (2) medical disorders that could affect CNS function (for example, impaired cardiopulmonary, renal, or hepatic function, hypothyroidism or hyperthyroidism, severe anaemia, vitamin $\mathrm{B} 12$ and folate deficiency, diabetes mellitus); (3) psychiatric disorders (past or present): in particular, depressive disorders (major depression or dysthymic disorder, according to the DSM-III-R criteria) which, in some cases, sustain an apparent dementing syndrome known as depressive pseudodementia. Clinical evidence of one of the above conditions led to exclusion, even if it was not considered to be the relevant cause of cognitive impairment in that particular patient.

In all the 24 patients MRI was performed also using transverse and coronal planes which allow a good visualisation of the mesial temporal cortex (hippocampal and parahippocampal gyri). In all the 24 patients there was a mild to moderate brain atrophy, consistently involving the hippocampal structures, in the absence of focal lesions attributable to vascular or other diseases. There were no clear cut differences between MRI pictures of patients successively classified as FTLD and dAD (see below ): the distribution of atrophic changes was not exclusively focal (mesial-temporal) in the first group and diffuse in the second one. Rather, there was a continuum in the severity of widespread brain atrophy from patients with FTLD and patients with $\mathrm{dAD}$ with mild to moderate cognitive impairment to patients with $\mathrm{dAD}$ with severe cognitive impairment.

NEUROPSYCHOLOGICAL ASSESSMENT

Patients and control subjects were initially tested with the mini mental state examination $(\mathrm{MMSE})^{6}$ and the mental deterioration battery (MDB).

Fourteen patients were classified as having $\mathrm{dAD}$ (eight men and six women; mean age 65 years; mean duration of illness 14 months) as they presented a diffuse and rather homogeneous profile of cognitive impairment involving all the cognitive domains. Ten patients were considered as affected by FTLD (five men and five women; mean age 67.3 years; mean duration of illness 16 months) as they showed a focal pattern of cognitive impairment characterised by deficits of memory (either verbal or non-verbal) and language (word generation with semantic constraints). All patients with FTLD and 10 out of 14 patients with dAD presented a mild to moderate cognitive impairment whereas the remaining four patients with $\mathrm{dAD}$ showed a severe cognitive impairment. Criteria for defining cognitive impairment as mild to moderate were the following: (a) a score $\geqslant 15$ and $\leqslant 23$ on the MMSE, (b) the ability to complete all the test of the MDB with at least one test performance within the normal range, according to a previously tested proce- 
dure. Patients with MMSE score $\leqslant 15$ and none of the test scores of the MDB within normal limits were considered as affected by a severe cognitive impairment. The 12 control subjects (seven men and five women; mean age 63.2 years) were found to be free from cognitive abnormalities at neuropsychological assessment.

The tasks forming the MDB with mean scores obtained by FTLD and dAD groups are shown in table 1. Data reported in the table indicate that patients with FTLD scored at the same pathological level of (or even worse than) patients with $\mathrm{dAD}$ on measures of episodic memory (immediate and delayed recall, delayed recognition of the auditory verbal learning test; digit span) and on word generation (semantic categories), whereas they scored better than patients with $\mathrm{dAD}$ (and above the cut off values) on all the other neuropsychological measures.
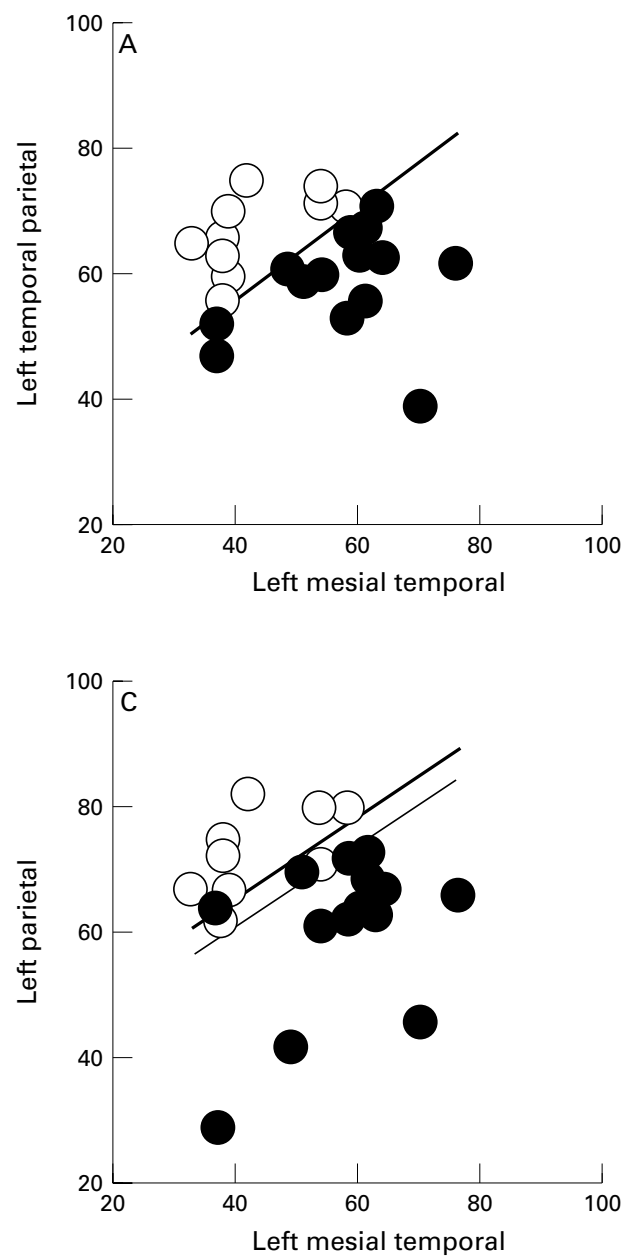

SPECT IMAGING PROCEDURES

All subjects were examined in an identical setting. Regional cerebral perfusion was evaluated using a four headed dedicated tomograph (CERTO 96, Selo, Italy), equipped with HR collimators. The tomographic system resolution at the centre of the transverse slice was 8.3 FWHM (with HR collimators) and the absolute point source sensitivity was $9.200 \mathrm{cpm} / \mu \mathrm{Ci}$ using ${ }^{99 \mathrm{~m}} \mathrm{Tc}$ (with GP collimators). A total of $740 \mathrm{MBq}\left[{ }^{99 \mathrm{~m}} \mathrm{Tc}\right]-\mathrm{HMPAO}$ (Amersham, UK) were administered intravenously. Image acquisition started 20 minutes after injection; total acquisition time was 30 minutes. Data were collected for 64 projections. Projection images were smoothed and reconstructed using a raised cosine filter, and then applying attenuation correction. Twenty eight transversal, coronal, and sagittal slices, each $7.5 \mathrm{~mm}$ thick, were obtained.
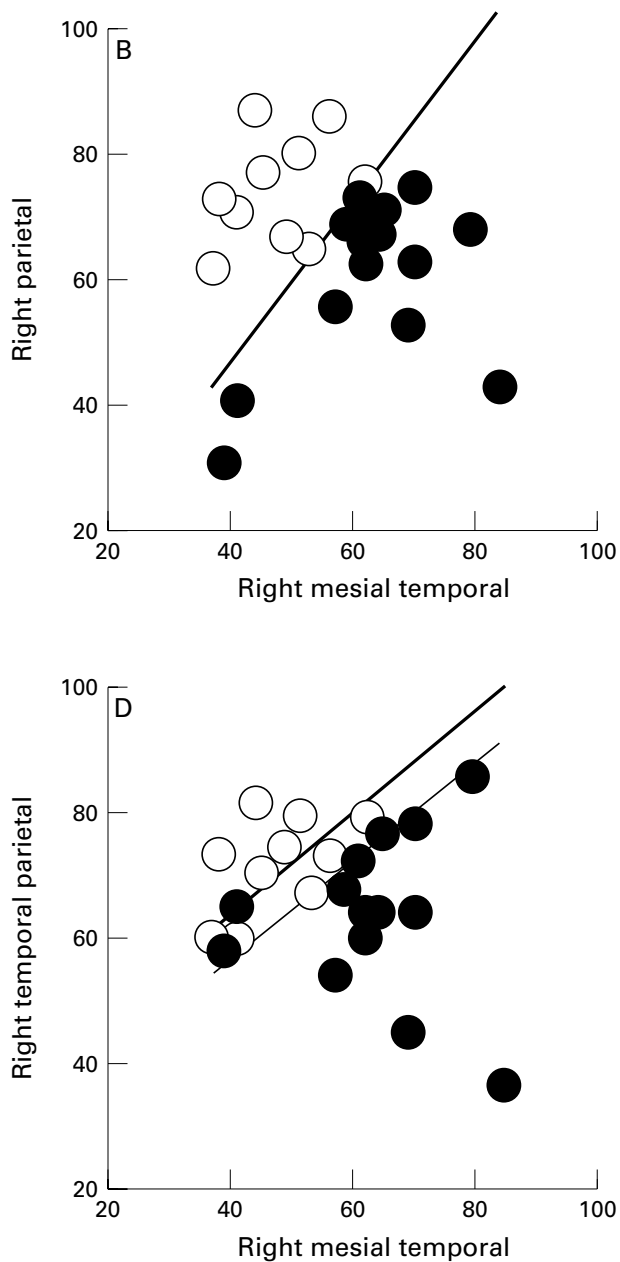

Figure 1 Predicting group membership of patients with $d A D$ and those with FTLD by different combined criteria: (A) Left mesial temporal and temporal parietal, (B) right mesial temporal and parietal, (C) left mesial temporal and parietal, (D) right mesial temporal and temporal parietal. In each figure $(A, B, C$, and $D)$ the space is divided in two areas-namely, the FTLD area (above the straight line) and the $d A D$ area (below the straight line). Each patient is represented as a point in this space and classified as FTLD if the point lies in the area above the straight line or as $d A D$ if the point lies in the area under the straight line (the dots represent the 24 patients included in the ROC analysis). In $C$ and $D$ two straight lines are plotted: by the thickest ones, the group membership of patients with dAD is correctly predicted (but that of patients with FTLD is missed in $20 \%$ and $50 \%$ of cases, respectively); by the thinnest ones patients with FTLD are correctly classified (but patients with dAD are misclassified, in both criteria, in $14.3 \%$ of cases). 
A semiquantitative evaluation of brain SPECT data was made considering the transversal slices (reoriented parallel to the orbitomeatal plane) and following the scheme of anatomically defined regions of interest (ROIs) proposed by Tran Dinh et al, ${ }^{8}$ with some additions. According to Tran Dinh et al, three bilateral ROIs (superior frontal, central, and parietal regions) were semiautomatically drawn on three to four consecutive vertex cerebral slices; six bilateral ROIs (anterior frontal, mid-
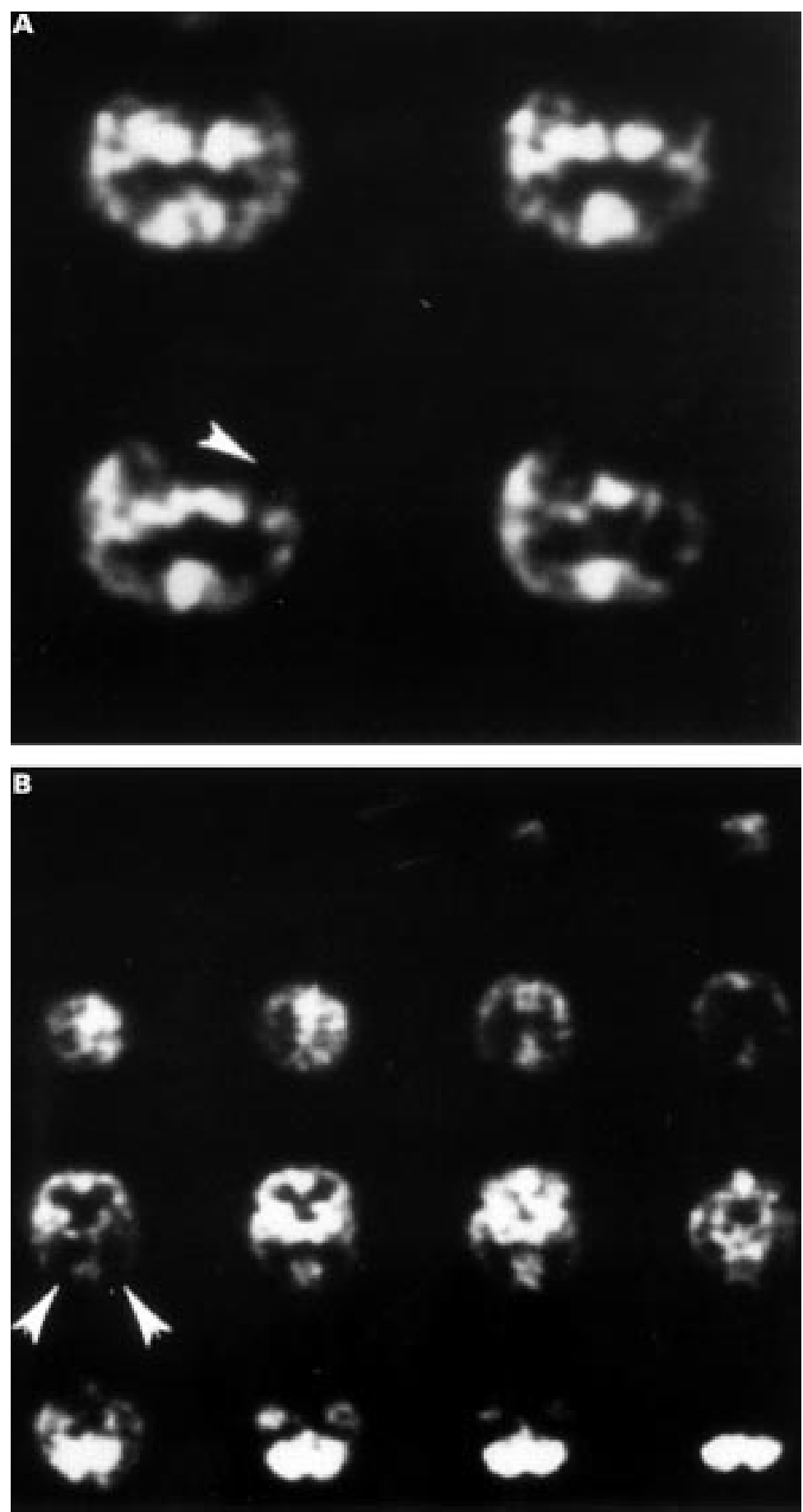

Figure 2 Representative [ [99m Tc]-HMPAO SPECT images of brain perfusion abnormalities in FTLD and patients with dAD. (A) Patient with FTLD: reduction of tracer uptake (arrow) in the left mesial and lateral temporal regions. (B) Patient with mild to moderate dAD: bilateral reduction of tracer uptake (arrows) in the temporal parietal areas. dle frontal, sylvian, temporoparietooccipital, occipital, and thalamic regions) were drawn on three to four consecutive mid-cerebral slices; two bilateral ROIs (anterior temporal region, emicerebellar region) and one middle ROI (pons) were drawn on two to three consecutive basal cerebral slices. To yield a satisfactory visualisation of the temporal structures, two transverse slices were also reconstructed along the major axis of the temporal lobe and two additional bilateral ROIs were drawn on the mesial and lateral temporal regions. Regional cerebral perfusion data were semiquantitatively computed by the ratio between each cerebral ROI and the cerebellar ROI with the highest counts.

\section{STATISTICAL ANALYSIS}

An analysis of variance (ANOVA) for repeated measures was carried out to test the effects of group (dAD, FTLD, CS), ROI, side (right and left hemispheres) and their interactions. The significance of the differences in $\mathrm{rCBF}$ for each ROI between pathological groups and controls were evaluated by Student's $t$ test for independent groups. Bonferroni's correction was used to control the probability of type I errors when performing multiple comparisons. Two criteria were adopted: the first one took into account both ROIs and groups (81 comparisons) with a level of sigificance of $\mathrm{p}=0.0006$ for each pairwise comparison. The second, a less conservative criterion, considered only the groups (three comparisons) with a level of significance of $\mathrm{p}=0.0167$ for each comparison. The last criterion is more appropriate in the case of exploratory small studies. ${ }^{9}$

To select SPECT patterns which significantly, and in the most effective way, differentiated patients with dAD and those with FTLD, a logistic regression analysis, followed by a receiver operating characteristic (ROC) analysis, was also performed.

\section{Results}

The analysis of variance (ANOVA) repeated measures showed significant interactions of side $\times$ ROI $\left(F_{12,264}=2.91\right)$ and ROI $\times$ group $\left(\mathrm{F}_{12 \text {, }}\right.$ $\left.{ }_{264}=7.38\right)$.

The mean counts in the 27 cerebral ROIs of control subjects, patients with FTLD, and patients with $\mathrm{dAD}$ are reported in table 2 .

The logistic regression and ROC analyses were performed separately for each hemisphere. In both hemispheres, the mesial temporal area was able to predict the group membership of patients and even more if this area was combined with other areas (parietal, occipital, superior frontal for the left hemisphere; temporal parietal occipital superior frontal central for the right hemisphere); however, the best classifications were obtained using the following combined criteria: left mesial temporal plus left temporal parietal areas, and right mesial temporal plus right parietal areas (fig 1).

Figure 2 shows representative SPECT images of patients with FTLD and patients with dAD. 


\section{Discussion}

Patients with $\mathrm{AD}$ who exhibit a pattern of cognitive impairment restricted to memory (either verbal or non-verbal) and language (lexical and semantic components) with no significant deficits in perception, construction, attention, or executive functions, have been described as having a focal temporal lobe dysfunction (FTLD) and considered as a distinct subgroup-perhaps a distinct biological variant-of $\mathrm{AD} .^{5}$

In the present study, a semiquantitative analysis of cerebral perfusion in FTLD and patients with $\mathrm{dAD}$ using [ $\left.{ }^{99 \mathrm{~m}} \mathrm{Tc}\right]-\mathrm{HMPAO}$ SPECT has pointed out that the two groups, identified on the basis of distinctive profiles of cognitive deficits, were quite different even in terms of SPECT images. In comparison with normal controls, patients with FTLD showed a significant reduction of tracer uptake in the right and left mesial temporal, left anterior temporal, left lateral temporal, and left central regions, whereas patients with $\mathrm{dAD}$ showed a pathological hypoperfusion in the right and left parietal, right and left temporal parietal, right and left central, right and left superior frontal, and left occipital regions.

The hypoperfusion of the mesial temporal regions (either right or left) was so relevant in patients with FTLD as to differentiate them not only from controls but even from patients with $\mathrm{dAD}$. On the other hand, the perfusion deficit of the left temporal parietal and of the parietal areas bilaterally was a distinctive pathological feature of the dAD group, as it was not present in the FTLD group. Thus two distinctive scintigraphic patterns, shaping a double dissociation between patients with FTLD and those with $\mathrm{dAD}$, emerged: the bilateral mesial temporal hypoperfusion, characteristic of the FTLD group, and the bilateral parietal and left temporal-parietal hypoperfusion, characteristic of the $\mathrm{dAD}$ group. Moreover, results of the logistic regression and ROC analyses pointed out that the combination of two SPECT patterns (mesial temporal and temporal parietal hypoperfusion in the left hemisphere and mesial temporal and parietal hypoperfusion in the right hemisphere) could correctly predict the FTLD or dAD group membership. However, studies on larger groups of patients are needed to validate such diagnostic criteria.

Our SPECT findings in patients with dAD confirm the currently accepted notion of a bilateral (either symmetric or asymmetric) hypoperfusion of the posterior temporal and parietal areas as the most typical SPECT picture in patients with $\mathrm{AD}$ with widespread cognitive deterioration. ${ }^{10}$ It is worth noting that, if the four patients with $\mathrm{dAD}$ with severe cognitive impairment were excluded from comparisons with controls, the left and right superior frontal, right central, and left occipital areas were no more involved in the pattern of significant perfusion deficits. This is consistent with the hypothesis that in AD brain damage spreads from the temporal parietal association cortex to the occipital and frontal cortex, according to the progression of disease severity. ${ }^{11}$

The mesial temporal pattern of SPECT abnormalities documented in the FTLD group fits the neuropsychological evidence of a selective deficit of episodic and semantic memory in these patients. As recently reported by Hodges and Patterson, ${ }^{12}$ episodic memory impairment in $\mathrm{AD}$ is sustained by lesions which first involve the transentorhinal region (causing disconnection of the hippocampus) and then the limbic structures (entorhinal cortex, subiculum, and hippocampal zone CA1) and the isocortical association areas. On the other hand, semantic memory impairment occurs when the pathology also extends to the adjacent temporal neocortex. A mesial temporal localisation of brain impairment was already reported by studies using SPECT in patients with early $\mathrm{AD}$ presenting amnesia as the main symptom. ${ }^{13}$ Patients with FTLD, however, are not likely to be patients in the early stages of the more common form of dAD (patients who have not yet exhibited perceptual, constructive, attentional, and executive dysfunction but whose disease course will follow the same trajectory as in $\mathrm{dAD})$. In our series, the estimated duration of disease at the time of evaluation was not significantly different in the FTLD and dAD groups. Moreover, the double dissociation that we found between the two patient groups in patterns of SPECT abnormalities is a strong argument in favour of the hypothesis, already advanced by Butters et al, ${ }^{5}$ that FTLD is a distinct variant of $\mathrm{AD}$. Data collected in the present study do not allow us to specifically address the question of whether we are dealing with an anatomoclinical or even a biological variant of AD. Preliminary results of a longitudinal study in progress - with serial neuropsychological assessments over a period of more than 2 years - at least confirm one of the main claims of the above cited study, ${ }^{5}$ showing that, in comparison with $\mathrm{dAD}$, patients with FTLD present a slower rate of cognitive decline.

In conclusion, results of this study provide further support to the hypothesis that FTLD is not a mere stage but rather represents a distinct form of $\mathrm{AD}$. Although the cognitive features of patients with FTLD-concerning the neuropsychological profile and the rate of cognitive decline-were already reported in the literature, the scintigraphic findings were never investigated and described. The present study shows that patients with FTLD can be distinguished from either controls or patients with $\mathrm{AD}$ with diffuse cognitive impairment even on the basis of distinctive patterns of SPECT abnormalities. The mesial temporal pattern of SPECT abnormalities documented in the FTLD group also denies the possible objection that, in our series, FTLD represented a group of patients misdiagnosed as $\mathrm{AD}$ and in reality affected by a circumscribed cortical degeneration - namely, Pick's disease. In Pick's disease brain damage evolving to atrophy generally involves the anterior portion of the frontal lobe and eventually the anterior portion of 
the first and second temporal gyri (T1, T2), but usually spares the temporal mesial structures such as the Ammon horn and the hippocampus. ${ }^{14}$ On the other hand, it must also be remembered that in the already cited series of 32 patients with FTLD reported by Butters et $a l,{ }^{5}$ the diagnosis of $\mathrm{AD}$ was confirmed at a pathological level in all the necropsied cases.

1 Martin A, Brouwers P, Lalonde F, et al. Towards a behavioural typology of Alzheimer's patients. F Clin Exp behavioural typology of Alzh
Neuropsychol 1986;8:594-610.

2 Jorm AF. Subtypes of Alzheimer's dementia: a conceptual analysis and critical review. Psychol Med 1985;15:543-53

3 Haxby JV, Grady CL, Koss E, et al. Longitudinal study of cerebral metabolic asymmetries and associated neuropsychological patterns in early dementia of the Alzheimer type. Arch Neurol 1990;47:753-60.

4 O'Brien JT, Eagger S, Syed GMS, et al. A study of regional blood flow and cognitive performance in Alzheimer's disease. $\mathcal{7}$ Neurol Neurosurg Psychiatry 1992;55:1182-7.

5 Butters MA, Lopez OL, Becker JT. Focal temporal lobe dysfunction in probable Alzheimer's disease predicts a slow rate of cognitive decline. Neurology 1996;46:687-92.
6 Folstein MF, Folstein SE, McHugh PR. Mini-mental state: a practical method for grading the cognitive state of patients for the clinicians. $\mathcal{F}$ Psychiatr Res 1975;12:189-98.

7 Carlesimo GA, Calatgirone C, Gainotti G, et al. Standardization of the mental deterioration battery. Eur Neurol 1996; 36:378-84.

8 Tran Dinh YR, Mamo U, Cervoni J, et al. Disturbances in the cerebral perfusion of human immune deficiency virus-1 seropositive asymptomatic subjects: a quantitative tomography study of 18 cases. 7 Nucl Med 1990;31:1601-7.

9 Davis C, Gaito J. Multiple comparison procedure within experimental research. Canadian Psychology 1984;25:1-13.

10 Perani D, Di Piero V, Vallar G, et al. Technetium -99m HM-PAO-SPECT study of regional cerebral perfusion in early Alzheimer's disease. F Nucl Med 1988;29:1507-14.

11 Frlich L, Eilles C, Ihl R, et al. Stage-dependent reductions of regional cerebral blood flow measured by HMPAOSPECT in dementia of Alzheimer type. Psychiatry Res 1989;29:347-50.

12 Hodges JR, Patterson K. Is semantic memory consistently impaired early in the course of Alzheimer's disease? Neuroanatomical and diagnostic implications. Neuropsychologia anatomical and

13 Ohnishi T, Hoshi H, Nagamachi S, et al. High-resolution SPECT to assess hippocampal perfusion in neuropsychiatric diseases. F Nucl Med 1995;36:1163-9.

14 Bugiani O, Mancardi GL. Demenze degenerative. In: Schiffer D, ed. Neuropatologia. Roma: Il Pensiero Scientifico, 1980:205-17. 